\title{
IATROGENIC URETERIC AND BLADDER INJURIES IN OBSTETRIC AND GYNAECOLOGIC SURGERIES
}

\author{
K. A. MTETA, J. MBWAMBO and M. MVUNGI
}

\begin{abstract}
Objective: To review iatrogenic ureteric and urinary bladder injuries from obstetric and gynaecological surgeries treated in the urology department analysing; ureteric anatomy, aetiologic factors, diagnosis, treatment and outcomes.

Design: A retrospective study.

Setting: Institute of Urology, Kilimanjaro Christian Medical Center (KCMC), Moshi, Tanzania. Subjects: Twenty three women were treated for iatrogenic ureteric and bladder injuries secondary to obstetric and gynaecological procedures in the department of urology between June 1994 and July 2004.

Results: Hysterectomy was the leading cause of ureteric injuries contributing to nine $(47.4 \%)$ of the 19 ureteric injuries. Caesarian sections were the second leading cause and contributed $6(31.6 \%)$ of 19 ureteric injuries. Vesical vaginal fistula (VVF) repairs lead to two (10.5\%) ureteric injuries one of which was bilateral. There was a case each $(5.3 \%)$ from ovarian cystectomy and forceps delivery. There were only three cases of intra-operative diagnosis of ureteric injuries. There were four bladder injuries half of which came from hysterectomy. The leading definitive urological treatment for ureteric injury was ureteric re implantation and all kidneys were saved.

Conclusion: Iatrogenic ureteric and bladder injuries from gynaecologic surgeries are globally rare but are liable to occur due to the inherent ureteric anatomic factors in the pelvis. Intra-operative diagnosis of injury is a rare feature. The practical principles to prevent and repair ureteric injuries have been presented and discussed. The true risk to the patient lies in delayed, missed diagnosis and inadequate treatment. Endourologic techniques offer an alternative diagnostic and treatment method for women previously injured in open pelvic surgeries.
\end{abstract}

\section{INTRODUCTION}

Surgical intervention responsible for ureteric injuries include total abdominal hysterectomy and bilateral salpingo ophrectomy followed by radical hysterectomy (Wertheim) and Caesarian section. Other rare causes are secondary to cervicocystopexies both pubic or vaginal, ovarian surgeries and uterine aspiration. The majority of injuries are secondary to abdominal surgeries.

In reports of studies not involving routine cystoscopy, the frequency of ureteral injuries varied from $0-14.6 \%$ injuries per 1000 operations. The frequency of bladder injuries varied from 0.2 to 19.5 per 1000, with an overall frequency of 2.6 per 1000. Only $11.5 \%$ of ureteral injuries and $51.6 \%$ bladder injuries were identified and managed intraoperatively (1).

In studies involving routine cystoscopy, the frequency of ureteral injury varied from 0 to 26.8 per 1000 with an overall frequency of 6.2 per 1000 . The frequency of bladder injury varied from 0 to 29.2 per 1000 , with an overall frequency of 10.4 per 1000. Up to $90 \%$ of unsuspected ureteral injuries and 
$85 \%$ of unsuspected bladder injuries were identified with the use of cystoscopy and were managed successfully intra-operatively. Operative injuries during vaginal hysterectomy are relatively rare. They are easily recognised and repaired during the primary operation without important sequelae. Rates of injuries secondary to laparoscopic procedures are not clearly known. Anatomic variation of the course of the ureters probably contribute to injuries in about $0.5 \%$ of cases (2).

The ureters enter the pelvic cavity as they cross the bifurcation of the common iliac artery. The uterine artery accompanies the ureter as it passes on the lateral lower aspect of the uterus and superior portion of the vagina. At the base of the cardinal ligament, the ureter passes over the uterine artery. At this zone it is often called "genou de l'uretere" because it changes its direction. At this point the ureter is about $12 \mathrm{~mm}$ from the wall of the vagina. As it passes to enter the base of the bladder it becomes even more close to the anterior wall of the vagina $(3,4)$.

\section{MATERIALS AND METHODS}

This was a retrospective study of consecutive patients with ureteral and urinary bladder injuries from obstetric and gynaecological interventions for benign conditions. This review involved patients referred and managed in the department of urology for a period of ten years from June 1994 to July 2004.

During this period, a total of 25 patients were referred to urology services of which five were from KCMC while 20 patients originated from hospitals in the KCMC catchments area.

Two patients were excluded from the study, one of which was a spontaneously vaginal delivery patient from KCMC who developed a vesical uterine fistula. The second was a patient referred from outside KCMC through gynaecological services to urology after a pelvic surgery whereby her undiagnosed pelvic horseshoe kidney was injured in an attempt to remove a "pelvic mass".

All 25 patients case notes were retrieved from the medical records and analysed in detail the patients name, age and name of the referring hospital. Further information was sought on, primary surgery done, intra-operative difficulties, post-operative complications and the genital urinary injuries suspected.
Patients suspected to have genital urinary injuries underwent, pre-treatment evaluations including: history taking and physical examination. Investigations done included: ultrasound of the urinary tract, serum creatinine and intra-venous pyelogram. Cystoscopy and vaginoscopy as well as retrograde ureteric catheterisation and contrast studies were done when appropriate. The aim of history taking, examination and investigations was to locate and delineate the injury so that surgical intervention can be planned. Definitive surgery to correct the injury was planned as soon as the patient was fit for anaesthesia and surgery. If severe hydronephrosis was present and sepsis or infection endangering renal function, a preliminary nephrostomy was done and definitive surgery was deferred to later date.

Definitive surgical interventions included, ureteric re-implantation, ureteric repair, transabdominal vesical vaginal fistula (VVF) repair and vesical uterine fistula repairs, foreign body removal as appropriate while patient follow up included; serum creatinine, ultrasound and IVP.

\section{RESULTS}

The 23 patients who were analysed are presented in this paper. The average age was 36.5 years (range 20-51 years). Further urology evaluation revealed that none of the patients had any demonstrable congenital anatomical abnormalities of the urinary system. Primary surgeries leading to the injuries were as follows: Total abdominal hysterectomies and bilateral salpingo ophrectomy (TAH+BSO) 11, Caesarian section seven, ovarian cystectomy two, VVF repair two, forceps delivery and or VVF repair two (Table 1).

$T A H+B S O$ : Under this category, there were 11 patients, one of which was from KCMC while the other ten were referred from peripheral hospitals. In total there were nine ureteric injuries and two bladder injuries.

There were two intra-operative diagnosis of ureteric injury, one of which was unsuccessfully repaired, while in the other patient, no attempt to repair was made. In the remaining nine patients, diagnosis was made during the post operative period following complications whereby seven patients with ureteric injuries presented with per vaginal urine 


\section{Table 1}

Primary surgeries leading to ureteric or bladder injuries

\begin{tabular}{lccccrc}
\hline \multirow{2}{*}{ Surgery } & \multicolumn{2}{c}{ Ureter } & \multicolumn{2}{c}{ Bladder } & \multicolumn{2}{c}{ Total } \\
& No. & $(\%)$ & No. & $(\%)$ & No. & $(\%)$ \\
\hline TAH+BSO & 9 & 50 & 2 & 40 & 11 & 47.8 \\
LSCS & 6 & 33.2 & 1 & 20 & 7 & 30.4 \\
ADNEXECTOMY & 1 & 5.6 & 1 & 20 & 2 & 8.7 \\
VVF REPAIR* & 2 & 8.7 & - & - & 2 & 8.7 \\
FORCEPS/VVF & 1 & 4.4 & - & - & 1 & 4.4 \\
\hline TOTAL & 19 & 100 & 4 & 100 & 23 & 100 \\
\hline
\end{tabular}

* One bilateral ligation of ureter and one metal probe foreign body in ureter

leakage, one patient presented with cutaneous urine leak from the surgical wound and one presented with acute lumbar pain later followed by per vaginal urine leakage. Apparently in all these surgeries, no intraoperative anatomical difficulties such as adhesions and or anatomical abnormalities were reported by the operating surgeons. In all the nine patients with ureteric injuries following hysterectomy, the injuries were in the lower third of the ureter within five centimeters of the bladder with either ligation and or sectioning of the ureter. Seven patients were successfully treated with ureteric re-implantation. The first of the patients diagnosed intra-operatively above, underwent successful ureterouretero anastomosis, two days after the primary surgery. The second patient diagnosed intra-operatively and repaired above, required a nephrostomy for severe hydronephrosis. This patient later underwent a definitive ureteric reimplantation with psoas hitch. In all the ureteric surgeries were done, a double "J" stent was left in situ to be removed six weeks later (Table 3 ).

There were two bladder injuries none of which were diagnosed intra-operatively and who presented with post operative per vaginal urine leakage. Both patients had trans-abdominal vesical vagina fistula repair successfully (Table 3 ).

Caesarian section: Under this category, there were seven patients all referred from outside KCMC of which six had ureteric injuries while one had bladder injury (Table 1).

In none of the cases, were the injuries diagnosed intra-operatively. In one patient the Caesarian section was described as difficult as there was a partial uterine rupture which required repair, while the other six patients the surgery was said to be "straightforward".
In six patients, the injury was on the left ureter while, only one injury occurred in the right ureter. With the exception of one patient who presented with cutaneous urine leak, the other six patients presented with post operative per vaginal urine leak.

All injuries to the ureter occurred in the lower third and involved ligation and or sectioning of the ureter.

In four cases ureteric re-implantation was done while in one case, an additional psoas hitch was required and in another patient, a simple ureteroureterostomy sufficed. There was one case of vesicouterine fistula which was successfully managed with trans abdominal fistula repair (Table 3 ).

Ovarian cystetomy: There were two patients (Table 1) one from outside KCMC in this category, one of whom had a right ovarian cystectomy and was noted to have per cutaneous urine leakage post operatively. Intraoperatively, multiple pelvic adhesions were reported. Urology evaluation revealed urinary ascites with demonstrable right ureter leak into the peritoneal cavity on IVP, in addition the patient had wound sepsis. The patient was resuscitated and stabilised and at exploration was found to have ligation and sectioning of the right lower third ureter. Right ureteric re- implantation was done (Table 3).

The second patient also underwent right ovarian cystectomy, pelvic adhesions were reported in the primary surgery and the patient had undergone an appendicectomy two years previously. She presented with haematuria during menses and investigations revealed a vesico uterine fistula of which a successful trans-abdominal repair was done (Table 3 ).

VVF repair: Two patients all of them from KCMC were in this group (Table 1). The first one developed 
Table 2

Clinical anatomic presentation of ureteric and bladder injuries

\begin{tabular}{lcccccccc}
\hline Surgery & $\begin{array}{c}\text { Uretero- } \\
\text { vaginal } \\
\text { fistula }\end{array}$ & $\begin{array}{c}\text { Uretero- } \\
\text { cutaneous } \\
\text { fistula }\end{array}$ & $\begin{array}{c}\text { Vesical- } \\
\text { uterine } \\
\text { fistula }\end{array}$ & $\begin{array}{c}\text { Vesico- } \\
\text { vaginal } \\
\text { fistula }\end{array}$ & $\begin{array}{c}\text { Anuria } \\
\text { bil. } \\
\text { ligation }\end{array}$ & $\begin{array}{c}\text { Uretero- } \\
\text { uterine } \\
\text { fistula }\end{array}$ & $\begin{array}{c}\text { FB in } \\
\text { ureter }\end{array}$ & Total \\
\hline TAH + BSO & 6 & 3 & - & 2 & - & - & - & 11 \\
LSCS & 4 & 1 & 1 & - & - & 1 & - & 7 \\
ADNEXECTOMY & - & 1 & 1 & - & - & - & 2 \\
VVF REPAIR & - & - & - & - & 1 & - & 1 & 2 \\
FORCEPS/VVF & 1 & - & - & - & - & - & - & 1 \\
\hline TOTAL & 11 & 5 & 2 & 2 & 1 & 1 & 1 & 23 \\
\hline
\end{tabular}

anuria and acute bilateral lumbar pains following trans-vaginal VVF repair. Exploration the next day revealed bilateral ligation of the ureters and bilateral ureteric re-implantation was necessary. The VVF repair remained intact however the patient leaked from an incompetent bladder sphincter despite a well healed VVF. She unsuccessfully underwent a bladder neck fascia sling and required a Mainz II pouch urinary diversion seven months later.

In the second patient who was undergoing a transvaginal VVF repair, the surgeon was using a metal probe to locate the ureter through the ureteric opening in the bladder. This metal probe accidentally slipped into the left ureter and could not be retrieved endoscopically. The VVF repair was left to heal and the offending metal probe had to be removed by open ureteric exploration two months later (Table 3).
Forceps delivery: In this category there was only one patient (Table 1) whose history is a little bit complicated. The patient underwent forceps delivery at a peripheral hospital after which she developed a VVF. She underwent VVF repair seven months later but continued to leak urine per vagina despite a well healed VVF repair. Further investigations revealed a left uretero vaginal fistula. It was not possible to establish with certainty as to the origin of this ureteric fistula, was it secondary to the forceps delivery or was it secondary to the subsequent VVF repair? A left ureteric reimplantation was done and the patient was discharged home continent (Table 3).

Table 3

Definitive urological treatment procedures done

\begin{tabular}{|c|c|c|c|c|c|c|c|}
\hline \multicolumn{8}{|l|}{ Surgery } \\
\hline & $\begin{array}{l}\text { Ureteric re- } \\
\text { implantation }\end{array}$ & $\begin{array}{l}\text { Ureteric re- } \\
\text { implantation + } \\
\text { psoas }\end{array}$ & $\begin{array}{c}\text { Ureteroure- } \\
\text { terostomy }\end{array}$ & $\begin{array}{l}\text { Vesicouterine } \\
\text { fistula } \\
\text { repair }\end{array}$ & $\begin{array}{l}\text { Vesicovaginal } \\
\text { fistula } \\
\text { repair }\end{array}$ & $\begin{array}{c}F B \\
\text { removal }\end{array}$ & Total \\
\hline $\mathrm{TAH}+\mathrm{BSO}$ & 7 & 1 & 1 & - & 2 & - & 11 \\
\hline LSCS & 4 & 1 & 1 & 1 & - & - & 7 \\
\hline ADNEXECTOMY & 1 & - & - & 1 & - & - & 2 \\
\hline VVF REPAIR & $1^{*}$ & & - & - & - & $1^{* *}$ & 2 \\
\hline FORCEPS/VVF & 1 & - & - & - & - & - & 1 \\
\hline TOTAL & 14 & 2 & 2 & 2 & 2 & 1 & 23 \\
\hline
\end{tabular}

* Bilateral ureteric re-implantation

** Metal probe foreign body in ureter removed by open surgery 


\section{DISCUSSION}

Ureteric injuries: In this series, hysterectomy was the most common cause of ureteric injuries and none of the procedures was reported to be a "difficult" one. All the injuries occurred in the lower third ureter most likely when ligating the uterine artery or when dissecting the lower uterus, cervix and upper vagina as reported in the literature cited. Intra-operative diagnosis was a rare feature and this concurs with the literature review.

In the LSCS group, also the most common injury was to the lower third of the ureters and in one case, the surgery is assumed to have been difficult as there was uterine rupture requiring repair. In the majority of the LSCS, the surgery was again "straight forward". There was no intra-operative diagnosis in this group. The mechanism of injury is probably that the ureter was involved in suture ligature of the lower uterine segiment or involved when sectioning the lower uterus if the incision extends too laterally. In both hysterectomy and LSCS, the ureter can be caught by sutures when re-peritonising.

Ureteric injuries has also been reported in ovarian cystectomy and the injury occurs during ligation of the mesoovary or during reperitonisation. The ureter may also be caught during ligation of the broad ligament of the ovary. Direct injuries may occur when clamping ovarian or uterine arteries especially when haemostasis was difficult to achieve (5).

To the authors' knowledge, literature on ureteric injuries during VVF repair in East Africa is rare or unavailable at best. In this series, we report one patient who unfortunately had bilateral ureteric ligation during VVF repair. One method of preventing this untoward event is by catheterising the ureters during VVF repair and in this series we report one patient whose ureter was being "sounded" with a metal probe which accidentally slipped beyond reach into the ureter. So far, the author is not aware of any similar incident reported in East African literature and the picture is the same for forceps delivery being complicated by ureteric injury.

Bladder injuries: There were a total of four bladder injuries, half of which came from hysterectomies and one each from LSCS and ovarian cystectomy. Most likely the injuries came as the bladder was being either sharply or bluntly dissected away from the lower uterus and cervix. It is a little bit difficult to explain the bladder injury when doing ovarian cystectomy, however if the ovarian cyst is grossly large and there are multiple adhesions requiring extensive dissection, bladder injury is liable to occur.

Other risk factors for ureteric injuries includes: previous interventions, pelvic inflammatory disease, endometriosis, anatomical anomalies and salpingoophrectomy before total abdominal hysterectomy. In two cases in this series significant pelvic adhesions were reported. Three quarters of ureteric injuries occur during gynaecological surgeries for benign conditions judged as "simple" by the practitioners, in this series the same scenario of "straight forward" surgeries was noted.

Precautionary measures: In KCMC, we advise that in order to have improved pelvic access during elective abdominal hysterectomy, the vagina should be tightly packed with an abdominal mop soaked in povidone iodine. This maneuver "lifts" the vagina and the cervix into the pelvis and therefore facilitates easier dissection of the lower uterus, cervix and upper vagina. We in urology do the same when we have to remove the bladder and uterus en block for carcinoma of the bladder and have found the maneuver quite useful.

The most important factor in prevention of ureteric injuries lies in adequate knowledge of pelvic anatomy and the common sites of ureteral lesions. It is imperative to visualise the ureters during pelvic dissection and to avoid as much as possible to devascularise the ureter. If the procedure is deemed to be difficult, it might be wise to identify and isolate the ureter in order to protect it.

In this series as in most other series cited, intraoperative diagnosis of injury is done in only a minority of the patients. In this study all kidneys were saved and no nephrectomies were necessary.

Immediate repair assures the best surgical results, if it is a ligature mishap it is released immediately, similarly for clamping with an artery forceps. If the ureter is sectioned, urine flowing into the operative field might be noted. In reality it is seldom that the majority of injuries are discovered intra-operatively. The majority of iatrogenic ureteric injuries are not suspected and intra-operative diagnosis is rare, this was the typical scenario in this study as well. 
Diagnosis: Two post operative circumstances should lead to suspicion of ureteric injuries and require rapid diagnostic evaluation: post operative anuria and leakage of urine cutaneously or vaginaly $(6,7)$.

In this study, this was the typical scenario with the majority of patients presenting with either cutaneous or vaginal leakage of urine.

In most cases post operative diagnosis is delayed, in some studies the delay ranges from 65 days to 10 years. The principal symptom is lumbar and or hypogastric pains, gastrointestinal discomfort and low grade non-specific fever, haematuria is absent or microscopic.

Deterioration of renal function can occur in the absence of significant lumbar pains. It is therefore necessary to have a high index of suspicion to diagnose ureteric injury, there may be a moderate elevation of serum creatinine levels.

Imaging is essential for diagnosis especially in cases where bilateral lesions are suspected. Ultrasound may show the eventual hydronephrosis, but this may not be obvious initially. Repeated ultrasound examinations may be necessary. Intravenous pyelogram is indispensable in delineating the degree and level of ureteric obstruction. Well draining cutaneous or vaginal fistulae may co-exist with partial ureteric obstruction or stenosis.

Post operative cystoscopy may be necessary in cáses where IVP does not show clear evidence of lesion. Cystoscopy and vaginoscopy may also identify vesico vaginal or vesical uterine fistulae. Retrograde ureteric catheterisation can be done at the same time and when successful can be therapeutic.

If a percutaneous nephrostomy catheter is in place, ante grade contrast studies can precisely delineate the site of ureteric obstruction or the path of fistulation. Immediate release of a suture ligature may suffice. Clamped or ligated ureteric segment should be assessed for devitalized tissues which should be excised.

Sectioned ureter can be repaired over a ureteric catheter and if the blood supply is adequate, healing is usually excellent. Upper ureteric injuries may require trans uretero anastomosis. Lower ureteric injuries in the vicinity of the bladder may require ureteric re-implantation with or without a psoas hitch.

Percutaneous nephrostomy is essential when ureteric injury and obstruction is associated with infection. In some cases nephrostomy is essential in order to preserve renal function when scarring and fibrosis at site of ureteric injury prevents immediate repair. A nephrostomy can also permit ante grade contrast studies, ureteroscopy and or ante grade ureleric catheterisation.

Percutaneous nephrostomy is not without complications some of which are: entry into peritoneal cavity, haemorrhage, perforation of viscera, and poor drainage. Nephrostomy may also be complicated by acute pyelonephritis while prolonged drainage without ureteric catheterisation ma! predispose to irreversible ureteric stenosis (8).

Management: Endoscopic urology has now a primary value in the treatment of ureteric lesions. Endoscopy can also permit ante-grade or retrograde ureteric catheterisation with or without prior ureteric dilatation. Ureteric catheters should be left in situ for at least six weeks. A guide wire can be passed endoscopically and can enable ureteroscopy to sucreed. Endoscopic incision of stenotic ureteral segment can be curable. In delayed diagnosis where fibrosis, scarring and epithelisation of fistula has taken place, endoscopic management is impossible (9).

Currently in KCMC almost all patients referred to urology with suspected genital urinary injury will undergo cystoscopy and retrograde ureteric catliterisation and contrast studies to delineate the ureleric injury.

Simple ureterolysis can relieve obstruction and restore anatomical continuity. Ureteric reimplantation is indicated for ureteric lesions which are within five centimetres of the uretero vesical junction, where re-implantation can be done without tension. The majority of patients $(64 \%)$ in this study were treated with re-implantation. (Table 3 ) In all these procedures, an antireflux mechanism is used commonly the Politano-Leadhetter method (10).

Occasionally when there is too much tension, a Boari flap is raised from the bladder to allow tension free anastomosis. The Boari flap is probably the method of choice for distal lesion. Its modification can create longer flaps which can be used even for upper ureteric injuries. A Psoas hitch may also be employed to achieve the same, where the bladder is hitched to the ipsilateral psoas muscle to allow a tension free re-implantation on the same side. Psoas hitch was used in three patients in this series.

Resection and uretero anastomosis with or without spatulation is suitable for the pelvic ureter. 
The technique is simple and is popular and effective among urologists. Mobilisation of the ureter should be done carefully to avoid de-vascularisation however periureteric fibrotic scar tissues should be excised. This technique was used in one patient only, the main reason being delayed presentation leading to urinomas and local fibrosis making it impossible to dissect and do anastomosis. (Table 3) Transuretero ureterostomie is contraindicated if the contralateral ureter is obstructed, in retroperitoneal fibrosis and in retroperitoneal tumours (11). McQuitty et al proposes bowel anastomosis with the inferior or superior calyces after partial polar nephrectomy (12).

\section{CONCLUSION}

Iatrogenic ureteric and bladder injuries from obstetric and gynaecologic surgeries are globally rare but are liable to occur due to the inherent anatomic and pathological factors in the pelvis.

Intra-operative diagnosis of ureteric and bladder injuries are a rare feature and late diagnosis in the post operative period is more common.

Effective diagnostic and therapeutic endourologic procedures are available to manage ureteric and bladder injuries for women previously injured in open pelvic surgeries. The practical principles to prevent and repair ureteric injuries have been presented and discussed.

The true risk to the patient lies in delayed, missed diagnosis and inadequate treatment. Modern imaging, treatment of infection and percutaneous nephrostomy have enabled preservation of renal function and assured a general good prognosis.

\section{ACKNOWLEDGEMENTS}

To the obstetricians and gynaecologists in $\mathrm{KCMC}$ and $\mathrm{KCMC}$ catchments area who referred the patients to urology department, giving us the privilege to manage these patients, the medical records department and hospital administration for allowing us to use hospital data for this study.

\section{REFERENCES}

1. Gilmour D.T., Dwyer P.L., and Carey M.P., Lower urinary tract injury during gynaecologic surgery and its detection by intra-operative cystoscopy. Obstet. gynecol. 1999; 95: 883-889.

2. Mathew P., Valencia P., Cousin C., et al. Operative injuries during vaginal hysterectomy. Eur. J. Obstet. Gynecol. Reprod. Biol. 2001; 97: 71-75.

3. Bruker L.T., and Wilbanks G.D. Urinary tract injuries in pelvic surgery. Surg. Clin. North Amer. 1991; 71: 963-976.

4. Cormio L. Ureteric injuries. Clinical and experimental studies. Scand. J. Urol. Nephrol. 1991; Suppl.171: 66p.

5. Neuman M., Eidelman A., Langer R., et al. latrogenic injuries to the ureter during gynaecologic and obstetric operations. Surg. Gynec. Obst. 1991; 173: 268-272.

6. Assimos D.G., Patterson L.C., and Taylor C.L. Changing incidence and etiology of iatrogenic ureteral injuries. J. Urol. 1994; 152: 2240-2246.

7. Falandry L. Les fistules urétéro vaginales: diagnostic et tactiques opératoires. A propos de 19 cas personnels J. Chir. 1992; 129: 306-319.

8. Turner W.H., Cranston D.W., Davies A.H., et al. Double $\mathrm{J}$ stems in the treatment of gynaecological injury to the ureter I. R. Soc. Med. 1990; 83: 623-624.

9. Blandy J.P., Badenoch D.A, Fowler C.G., et al. Early repair of iatrogenic injury to the ureter or bladder after gynaecological surgery. J. Urol. 1991; 146: 761-765.

10. Hodges C.V., Barry J.M., Fuchs E.F., et al. Transureteroureterostomy: 25 year experience with 100 patients. J. Urol. 1980; 123: 834-938.

11. Lask D., Arbanel J., Luttwak Z., et al. Changing trends in the management of iatrogenic ureteral injuries.

J. Urol. 1995; 154: 1693-1695.

12. McQuitty D.A., Boone T.B., and Preminger G.M. Lower pole calicostomy for the management of iatrogenic ureteropelvic junction obstruction. J. Urol. 1995; 153: 142-145. 